\title{
Influência do uso de lama de vidro na difusividade térmica de argamassas estruturais utilizando termografia
}

\author{
Influence the use of glass sludge on the thermal \\ diffusivity of structural mor-tars using thermography
}

\author{
Cristiane do Bom Conselho Sales Alvarenga ${ }^{1}$, \\ Oneida Heiderick Maximo ${ }^{3}$, Isabella Luísa Vieira Aquino Cassimiro ${ }^{3}$, \\ Rosemary do Bom Conselho Sales ${ }^{2}$, Maria Teresa Paulino Aguilar ${ }^{3}$
}

\footnotetext{
${ }^{1}$ Universidade Federal de Minas Gerais - DEMEC/DEES/ UFMG, CEP: 31270-901, 3409-1981, Belo Horizonte, MG, Brasil.

${ }^{2}$ Universidade do Estado de Minas Gerais - DEPC/UEMG, CEP: 31270-010, 3439-6519, Belo Horizonte, MG, Brasil.

${ }^{3}$ Universidade Federal de Minas Gerais - DEMC/UFMG, CEP: 31270-901, 3409-1981, Belo Horizonte, MG Brasil. e-mail: crisbcs@gmail.com, rosemary.sales@uemg.br, oneidaheiderick@ hotmail.com, isabellaluisa.aquino@fcagroup.com, teresa@ufmg.br
}

\section{RESUMO}

O vidro soda-cal-sílica é um material com alto potencial de reciclagem. No entanto, o seu beneficiamento gera micropartículas que ficam suspensas na água utilizada para resfriamento das ferramentas. Esse resíduo é inerte e, comumente, não reaproveitado. Para seu descarte é feita a decantação das micropartículas de vidro utilizando floculantes, sendo a lama produzida depositada em aterros sanitários. De acordo com a literatura, essas micropartículas de vidro poderiam atuar como material cimentício suplementar na produção de cimento Portland. Contudo, as informações sobre o desempenho térmico desses materiais são escassas. Neste estudo investiga-se a difusividade térmica de argamassas para fins estruturais confeccionadas com cimento Portland e lama de vidro soda-cal-sílica com características pozolânicas. Foram produzidas argamassas com e sem substituição do cimento por 10 e $20 \%$ de lama in natura e lama lavada para retirada do floculante. As argamassas foram caracterizadas quanto à resistência mecânica e difusividade térmica. Também, foram determinadas a massa específica, a condutividade térmica e o perfil de aquecimento das amostras, parâmetros necessários para avaliar a difusividade térmica. Os resultados indicam que a lama de vidro, com e sem floculante, poderia ser utilizada em substituição ao cimento sem comprometer a resistência mecânica devido às suas características pozolânicas. Nas argamassas com 10\% de lama sem floculante se observa um aumento de aproximadamente $8 \%$ na resistência. Contudo, em todas as amostras com lama de vidro, lavada ou não, observa-se uma diminuição de 3 a $4 \%$ na massa específica e de 17 a $18 \%$ na condutividade térmica, indicando que a reação pozolânica não ocorreu em toda a sua extensão. A difusividade, determinada com auxílio da termografia infravermelha, apresenta resultados compatíveis com a literatura e indica que o uso do resíduo não influencia significativamente a inércia térmica das argamassas. Dessa forma, a lama de vidro em substituição a $10 \%$ e $20 \%$ de cimento Portland poderia ser utilizada em argamassas estruturais sem comprometer o desempenho térmico do sistema, além de contribuir com o meio ambiente ao dar destino adequado ao resíduo e reduzir a retirada de matérias primas para a produção de cimento.

Palavras-chave: lama de vidro soda-cal-sílica; difusividade térmica; termografia infravermelha.

\section{ABSTRACT}

Soda-lime-silica glass is a material with high recycling potential. However, its processing generates microparticles that are suspended in the water used for cooling the tools. This residue is inert and generally not reused. For disposal, the microparticles of glass are decanted using flocculants and the mud produced is deposited in landfills. According to the literature, these glass microparticles could act as supplementary cementitious material in Portland cement production. However, information on the thermal performance of 
these materials is scarce. This study investigates the thermal diffusivity of mortars for structural purposes made with Portland cement and pozzolanic soda-lime-silica glass mud. The mortars were produced with and without cement replacement for 10 and $20 \%$ natural mud and washed mud to remove the flocculant. The mortars were characterized for compressive strength and thermal diffusivity. The specific mass, the thermal conductivity and the heating profile of the mortars were also determined, requirements to evaluate the thermal diffusivity. The results indicate that glass mud with and without flocculant could be used in place of cement without compromising compressive strength due to its pozzolanic characteristics. In mortars with $10 \%$ mud without flocculant an increase of approximately $8 \%$ in compressive strength is observed. However, in all samples with glass mud, washed or not, there is a decrease of 3 to $4 \%$ in the specific mass and 17 to $18 \%$ in the thermal conductivity, indicating that the pozzolanic reaction did not occur to its full extent. Diffusivity, determined with the aid of infrared thermography, presents results compatible with the literature and indicates that the use of the residue does not significantly influence the thermal inertia of the mortars. Thus, glass mud in place of $10 \%$ and $20 \%$ Portland cement could be used in structural mortars without compromising the thermal performance of the system, in addition to contributing to the environment by providing adequate destination to the waste and reducing the withdrawal of raw materials for cement production.

Keywords: soda-lime-silica glass sludge, thermal diffusivity, thermography.

\section{INTRODUÇÃO}

Uma das principais funções do espaço construído é prover ao usuário segurança e proteção contra intempéries, proporcionar conforto acústico e térmico no seu interior. Os materiais que constituem os fechamentos e envoltórias externas das edificações contribuem fortemente para esse conforto, que por sua vez está intimamente relacionado ao consumo de energia das edificações. Dessa forma a caracterização térmica dos elementos que os compõem pode contribuir para um melhor entendimento da capacidade de armazenamento e condução de calor em tais materiais, o que contribui para a avaliação do desempenho térmico dos edifícios.

Segundo FERRARI e ZANOTTO [1] e NBR 15220 [2], a transmitância/condutividade de materiais de construção pode ser estimada pelo uso do método da placa quente protegida ou pelo método fluximétrico. A condutividade térmica fornece a taxa de energia térmica transferida através de um corpo, como resultado de um gradiente de temperatura, sendo um indicativo da capacidade que o material possui de conduzir calor. No entanto, ela não fornece informações sobre a capacidade do material em armazená-lo. Dessa forma o desempenho térmico seria também expresso pela difusividade térmica, que é a propriedade relacionada à capacidade que o material possui de conduzir energia térmica em relação à sua capacidade de armazená-lo, ou seja, descreve quão rapidamente um material reage às mudanças de temperatura [3].

Um dos métodos para avaliação da difusividade térmica em regime transiente exige o conhecimento do perfil de temperatura do material. Tal perfil pode ser determinado por termografia infravermelha. Essa técnica permite mensurar a temperatura de diferentes tipos de materiais em pequenas e grandes áreas sem contato direto, apenas pela captação da energia térmica emitida pela superfície. A técnica está sendo utilizada em diferentes investigações onde o fenômeno de transferência de calor está envolvido [4]. Diferentes estudos relatam o uso de termografia para caracterizar e determinar a difusividade térmica dos materiais [5, 6]. No entanto, não é comum encontrar trabalhos sobre o uso da termografia para o estudo das propriedades térmicas de materiais de construção civil [7]. Trabalho de BARBOSA et al. [8] propõe uma técnica experimental alternativa para medição da difusividade térmica de blocos cerâmicos e de concreto com auxílio da termografia.

Os compósitos de cimento Portland são materiais largamente empregados na construção civil. Em função dos impactos ambientais gerados pela indústria cimenteira diferentes resíduos industriais têm sido utilizados como agregados ou em substituição parcial ao cimento $[9,10]$. Dentre esses resíduos destacam-se os resíduos de vidro soda-cal-sílica, que são inertes e podem ser reutilizados na fabricação de novos vidros. No entanto, no processo de corte, furação ou polimento do vidro são geradas nano e micropartículas que ficam em suspensão na água utilizada no resfriamento das ferramentas. Esse resíduo, em função da dificuldade de manuseio, não é reutilizado, e se disposto em rios provoca assoreamento, elevação do pH e turbidez da água, por não ser biodegradável [11]. Dessa forma faz-se necessário sua decantação com uso de floculantes. Como resultado do processo obtém-se uma lama impregnada de micropartículas de vidro que é usualmente depositada em aterros sanitários após decantação.

De acordo com a literatura, micropartículas obtidas por moagem de resíduos de vidro soda-cal-sílica poderiam atuar material cimentício suplementar, com a vantagem de inibirem a reação deletéria álcaliagregado [12,13]. Os vidros, no entanto, apresentam condutividade e difusividade diferentes das do cimento $[3,14]$, o que poderia influenciar o desempenho térmico dos compostos cimentícios produzidos com resíduos de vidro.

Considerando que são escassos os trabalhos sobre o uso de lama de vidro e sua influência no desem- 
penho térmico de compostos de cimento Portland, este trabalho tem como objetivo determinar a difusividade térmica de argamassas para fins estruturais confeccionadas com resíduo de vidro soda-cal-sílica em substituição parcial ao cimento Portland, utilizando termografia infravermelha.

\section{MATERIAIS E MÉTODOS}

Neste trabalho é analisado o desempenho térmico de argamassas para fins estruturais (concreto sem brita ou microconcreto) produzidas com e sem substituição parcial do cimento Portland por micropartículas de vidro soda-cal-sílica. O desempenho térmico das argamassas foi avaliado por meio de uma adaptação do método da placa quente protegida descrito na NBR 15220 [2] e por termografia infravermelha ativa (aquecimento artificial). As argamassas analisadas foram confeccionadas com cimento Portland de alta resistência inicial, água potável, areia industrial e resíduos de vidro contidos em lama proveniente do beneficiamento de vidro sodacal-sílica. A areia utilizada, oriunda da trituração do gnaisse (dimensão máxima característica 6,30 mm, massa especifica $2.620 \mathrm{~kg} / \mathrm{m}^{3}$ ) foi lavada para a retirada do excesso de finos e separada em faixas granulométricas (peneiras 1,2 mm, 0,6 mm, 0,3 mm e 0,15 mm). Para produção das argamassas utilizou-se a mesma quantidade de cada faixa granulométrica. Na Tabela 1 são apresentados os resultados da caracterização física e química do cimento utilizado.

Tabela 1: Caracterização do cimento Portland .

\begin{tabular}{c|c|l|c}
\hline \multicolumn{2}{c|}{ COMPOSIÇÃO QUímICA (\%) } & ENSAIOS & RESULTADOS \\
\hline $\mathrm{SiO}_{2}$ & 19,43 & Perda ao fogo $1000^{\circ} \mathrm{C}(\%)$ & 3,53 \\
\hline $\mathrm{Al}_{2} \mathrm{O}_{3}$ & 5,01 & Resíduo Insolúvel $(\%)$ & 0,83 \\
\hline $\mathrm{Fe}_{2} \mathrm{O}_{3}$ & 2,89 & Blaine $\left(\mathrm{cm}^{2} / \mathrm{g}\right)$ & 4771 \\
\hline $\mathrm{CaO}$ & 63,32 & Massa específica $\left(\mathrm{g} / \mathrm{cm}^{3}\right)$ & 3,11 \\
\hline $\mathrm{MgO}$ & 0,76 & Expansibilidade $(\mathrm{mm})$ & 0 \\
\hline $\mathrm{SO}_{3}$ & 3,00 & Início de pega $(\mathrm{min})$ & 131 \\
\hline $\mathrm{CO}_{2}$ & 2,63 & Final de pega (min) & 185 \\
\hline $\mathrm{K}_{2} \mathrm{O}$ & 0,79 & Resistência a compressão 1 dia $(\mathrm{MPa})$ & 30,3 \\
\hline \multicolumn{2}{l}{} & Resistência a compressão 28 dias $(\mathrm{MPa})$ & 55 \\
\hline
\end{tabular}

A lama de vidro utilizada é proveniente da furação, corte e polimento de vidros. Foi coletada na Estação de Tratamento de Efluentes de uma empresa beneficiadora de vidros. A lama, de alta viscosidade, continha vidros soda-cal-sílica de diferentes composições químicas, e floculantes. Estes floculantes são adicionados à água de resfriamento das ferramentas de corte/perfuração/polimento de forma a facilitar a decantação das micropartículas de vidro em solução, e dessa forma são incorporados aos resíduos. A lama, após a coleta, foi seca em estufa por 48 horas à temperatura de $105^{\circ} \mathrm{C}$. Em seguida foi destorroada e separada (fração passante na peneira $0,15 \mathrm{~mm}$ ). Parte do material passante foi utilizado para confecção das argamassas (argamassa com lama de vidro) e a outra parte foi submetida à lavagem com água para a retirada do floculante. O processo de lavagem foi repetido até a eliminação do floculante, que foi detectada pela coloração e odor da água. Essa operação foi necessária, pois, de acordo com SALOMÃO et al. [15], a contaminação da lama com floculante pode causar eflorescência nos corpos de prova de compostos de cimento Portland. Depois deste procedimento, o resíduo lavado foi novamente seco em estufa por 24 horas, a uma temperatura de $105^{\circ} \mathrm{C}$, e então utilizado na confecção das argamassas (argamassa com lama de vidro lavada). A caracterização física e química do resíduo de vidro antes e após lavagem é apresentada na Tabela 2.

Tabela 2: Caracterização do vidro soda-cal-sílica obtido por secagem antes após retirada do floculante. 


\begin{tabular}{|c|c|c|c|c|c|}
\hline Óxidos & $\begin{array}{l}\text { Lama de } \\
\text { vidro }\end{array}$ & $\begin{array}{c}\text { Lama de vidro } \\
\text { lavada }\end{array}$ & Partículas (\%) & Lama de vidro & Lama de vidro lavada \\
\hline $\mathrm{SiO}_{2}$ & 59,33 & 58,16 & 10 & 3,23 & 3,75 \\
\hline $\mathrm{CaO}$ & 34,99 & 36,31 & 50 & 16,39 & 15,15 \\
\hline $\mathrm{Fe}_{2} \mathrm{O}_{3}$ & 3,88 & 3,73 & 90 & 46,25 & 44,13 \\
\hline $\mathrm{K}_{2} \mathrm{O}$ & 0,48 & 0,55 & Diâmetro médio & 21,03 & 19,92 \\
\hline $\mathrm{SO}_{3}$ & 0,37 & 0,30 & & & \\
\hline $\mathrm{CuO}$ & 0,22 & 0,18 & \multicolumn{3}{|c|}{ CHAPELLE MODIFICADO } \\
\hline $\mathrm{SrO}$ & 0,15 & 0,12 & $\mathrm{mg}$ de $\mathrm{Ca}(\mathrm{OH})_{2} / \mathrm{g}$ & 587 & 559 \\
\hline $\mathrm{ZnO}$ & 0,79 & 0,05 & & & \\
\hline $\mathrm{MnO}_{2}$ & - & 0,05 & \multicolumn{3}{|c|}{ MASSA ESPECÍFICA } \\
\hline $\mathrm{Rb}_{2} \mathrm{O}$ & 0,03 & 0,03 & $\mathrm{~g} / \mathrm{cm}^{3}$ & 2,50 & 2,47 \\
\hline
\end{tabular}

Utilizou-se resíduo de vidro com e sem lavagem em substituição a 10 ou $20 \%$ de cimento Portland para produção das argamassas. A proporção utilizada foi de 1 parte de cimento para 3 partes de areia e fator água/aglomerante de 0,5. Considerando que os resíduos de vidro apresentam atividade pozolânica no Ensaio Chapelle Modificado [16], no cálculo da relação água aglomerante, considerou-se como aglomerante o cimento e o resíduo de vidro. De forma a não comprometer a trabalhabilidade, manteve-se constante o volume de aglomerante, isto é, a massa de resíduo equivalente a 10 e $20 \%$ de cimento foi calculada considerando-se a massa específica do vidro e cimento.

Após a moldagem de corpos cilíndricos de $5 \mathrm{~cm}$ diâmetro por $10 \mathrm{~cm}$ de altura, foram realizados ensaios de massa específica aparente e resistência mecânica à compressão. Para os ensaios de condutividade térmica e termografia foram utilizadas placas de $22,5 \times 11 \times 5,5 \mathrm{~cm}$. As amostras foram curadas em câmara úmida por 28 dias.

A massa específica aparente foi avaliada por determinação da massa em balança hidrostática com capacidade máxima de $32.000 \mathrm{~g}$ e resolução de $0,1 \mathrm{~g}$. Foram produzidas 2 amostras para cada tipo de argamassa.

Os ensaios de resistência à compressão foram realizados em uma prensa hidráulica da marca EMIC, modelo DL30000N, com sistema computadorizado de controle de aplicação de carga $(0,8 \mathrm{MPa} / \mathrm{s})$ e de aquisição de dados (software Tesc versão 3.04), com base na NBR 12142 [17]. Foram produzidas 6 amostras para cada tipo de argamassa.

A difusividade das argamassas foi estimada considerando o modelo matemático de transferência de calor unidimensional em regime transiente para um sólido semi-infinito. Segundo a literatura clássica, qualquer sólido com dimensões finitas pode ser considerado como um sólido semi-infinito desde que o distúrbio de temperatura em uma face não atinja a face oposta no intervalo de tempo considerado [3]. No presente estudo, para a aplicação dessa hipótese, a variação mínima de temperatura na face oposta foi considerada quando o valor ultrapassou a incerteza de medição. A Equação 1 descreve o modelo.

$$
T(x, t)-T_{i}=\frac{2 q_{0} \sqrt{\frac{\alpha t}{\pi}}}{k} e\left[-\frac{x^{2}}{4 \alpha t}\right]-\frac{q_{0}}{k} \operatorname{erfc}\left[\frac{x}{2 \sqrt{\alpha t}}\right]
$$

em que $T(x, t)$ representa a temperatura ${ }^{\circ} \mathrm{C}, T i$ a temperatura da superfície identificável no instante inicial ${ }^{\circ} \mathrm{C}$, qo o fluxo de calor constante aplicado na superfície identificável $\mathrm{W} / \mathrm{m}^{2}, \alpha$ a difusividade térmica do sólido $\mathrm{m}^{2} / \mathrm{s}$, $t$ o tempo s, $k$ a condutividade térmica do sólido W/m.K, $x$ a posição $\mathrm{m}$ e $\operatorname{erfc}$ () a função Erro de Gauss. Para a determinação da variação de temperatura ao longo das amostras em função do tempo utilizou-se a metodologia proposta por BARBOSA et al. [8] com base em ensaios termográficos.

Para os testes foi feita uma caixa térmica (Figura 1) com abertura suficiente para conter uma amostra da argamassa em estudo. Para que fosse possível medir o fluxo de calor, a caixa térmica foi vedada para impedir a perda de energia térmica para o ambiente. A caixa foi revestida internamente com placa isolante AT $1200 \mathrm{com} 25,4 \mathrm{~mm}$ da Texfiber e as amostras foram posicionadas na parte frontal da caixa conforme mostrado esquematicamente na Figura 1. Dentro da caixa o fluxo de calor foi constante, gerado por uma lâmpada 
cilíndrica halógena $127 \mathrm{~V}$ de $500 \mathrm{~W}$ de potência, colocada ao fundo da caixa. A parte superior (tampa) foi fixada no corpo da caixa por dobradiças. A radiação térmica superficial das amostras foi captada por termografia infravermelha - câmera Flir Systems, modelo SC660. Para a aquisição das imagens a câmera foi posicionada a uma distância de $0,80 \mathrm{~m}$ da parte frontal da caixa térmica e as medidas foram realizadas a cada $10 \mathrm{~s}$.
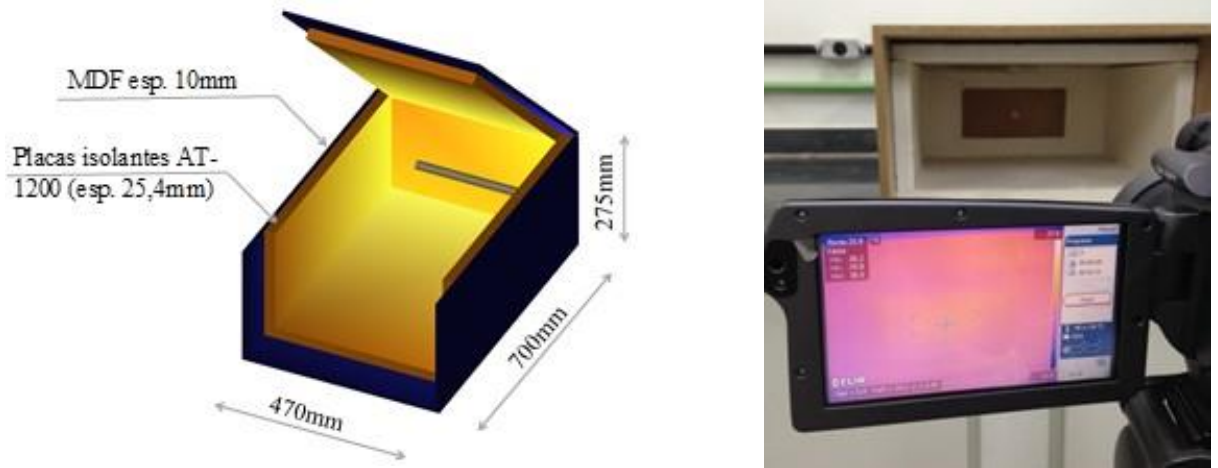

Figura 1: Esquema da caixa térmica e posição da termocâmera em relação à bancada experimental.

Para aquisição das imagens termográficas foi necessário a inserção de dados de entrada no equipamento, ou seja, a emissividade do material, a temperatura atmosférica, umidade relativa do ar e distância entre a câmera e a amostra.

A temperatura e a umidade do ar foram registradas pelo Relógio Termo Higrômetro Testo 622 e a distância da câmera até a amostra foi aferida com uma trena metálica. A emissividade da amostra foi determinada pela técnica de emissividade conhecida (fita crepe). A fita foi fixada no centro da amostra (que deve ficar em equilíbrio com o material em teste) e registrou-se um termograma com ajuste da emissividade da fita crepe para obter-se a temperatura de referência. Em seguida a emissividade foi sendo ajustada até que a câmera medisse a mesma temperatura na área sem a fita, obtendo-se assim o valor da emissividade térmica do material. Os valores de temperatura superficial obtidos pela termografia em função do tempo foram utilizados como dados de entrada para a Equação (A), e a difusividade $\alpha$ foi a variável de saída.

O fluxo de calor que incide ao longo da amostra $q x$ é um outro parâmetro necessário para o cálculo da difusividade térmica do material. Considerando o método utilizado (aquecimento por lâmpada halógeno), este fluxo pode ser calculado pela Equação 2.

$$
q_{x}=F_{i j} \frac{P o t}{A}
$$

onde $q x$ é o fluxo de calor $\mathrm{W} / \mathrm{m}^{2}, F i$-j é o fator de forma entre a superfície da lâmpada e da amostra, Pot é a potência da lâmpada $\mathrm{W}, A$ é a área da amostra $\mathrm{m}^{2}$. O fator de forma do fluxo de calor proveniente da lâmpada, considerada um cilindro infinitamente longo e paralelo à amostra (largura finita), foi calculado pelo programa Engineering Equation Solver.

Para o cálculo da difusividade térmica também se faz necessário conhecer a condutividade térmica, que foi determinada considerando o fluxo de calor através da placa. Conhecido o perfil de temperatura, a condutividade térmica e o fluxo de calor calculou-se a difusividade térmica das argamassas (Equação 1) utilizando o programa Engineering Equation Solver.

A análise da variância (ANOVA - one way) e o teste Tukey, calculados pelo aplicativo PAST versão 4.02, foram utilizados para avaliar comparativamente os valores obtidos nos diferentes ensaios, de forma a se identificar a influência do uso resíduo de vidro nas propriedades analisadas.

\section{RESULTADOS E DISCUSSÃO}

Na Figura 2 são apresentados os valores médios da resistência à compressão uniaxial das amostras com idade de 28 dias. A análise da variância indicou serem significativas as diferenças entre as médias das argamassas com vidro, e entre elas e a amostra de referência. O teste Tukey identificou que as possíveis dispersões entre as médias se relacionam ao valor da argamassa com 10\% de lama de vidro lavada: a substituição de $10 \%$ de cimento Portland por resíduo de vidro lavado contribui para uma leve melhoria ( $8 \%)$ da resistência à compressão em relação ao valor da amostra de referência, ou seja, o uso de resíduo de vidro nas condições estudadas não compromete a resistência à compressão das argamassas. Estudo de PEREIRA et al. [18] com cinzas pozolânicas mostra que as dosagens com melhores resultados de ganho de resistência aos 28 dias estão entre 5 e $10 \%$ de substituição ao cimento Portland. Por outro lado, diferentes pesquisas indicam que o uso de 10 e $20 \%$ de micropartículas de vidro soda-cal-sílica, isento de impurezas, em substituição ao cimento não 
afeta significativamente a resistência mecânica à compressão devido a atividade pozolânica das micropartículas $[12,19,20,21]$. O leve aumento ou a manutenção do valor de resistência à compressão observados neste estudo estariam associados à pozolanicidade do resíduo de vidro (Tabela 2). Nas amostras com 20\% de resíduo lavado o aumento não foi observado, provavelmente, devido ao efeito de dissolução do cimento e da menor disponibilidade de hidróxido de cálcio para a ocorrência da reação pozolânica. No caso das argamassas com resíduo não lavado, a não ocorrência do aumento da resistência à compressão seria devido à aglutinação das micropartículas de vidro promovida pela ação do floculante e, também, no caso dos $20 \%$, ao efeito de dissolução do cimento, efeitos que influenciam na extensão da reação pozolânica.

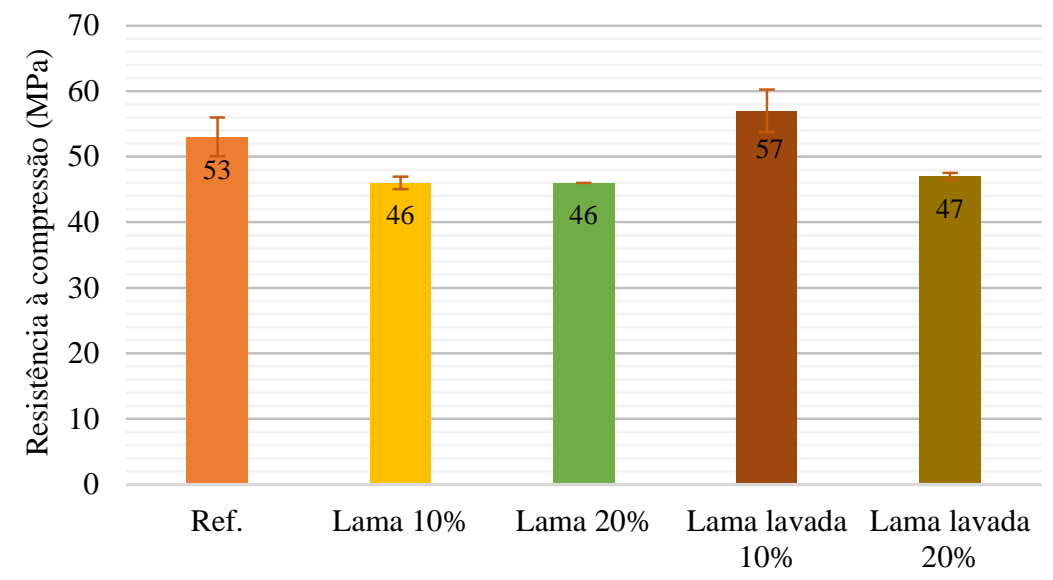

Figura 2: Resistência à compressão das argamassas com e sem lama de vidro.

Os valores médios das massas especificas das argamassas para fins estruturais são apresentados na Figura 3. A análise estatística das médias indicou não haver variação significativa entre os valores de massa específica das argamassas com resíduos de vidro. No entanto, identificou diferença entre os valores da argamassa de referência e das com lama de vidro. Nesse caso, o uso de lama de vidro, lavada ou natural, em substituição ao cimento promove uma ligeira diminuição da massa específica. Efeito que estaria relacionado à existência de vazios na microestrutura indicando que as reações pozolânicas não ocorreram em toda a extensão devido à idade das argamassas [22, 23] e/ou, também, aos efeitos de dissolução e aglutinação acima mencionados.

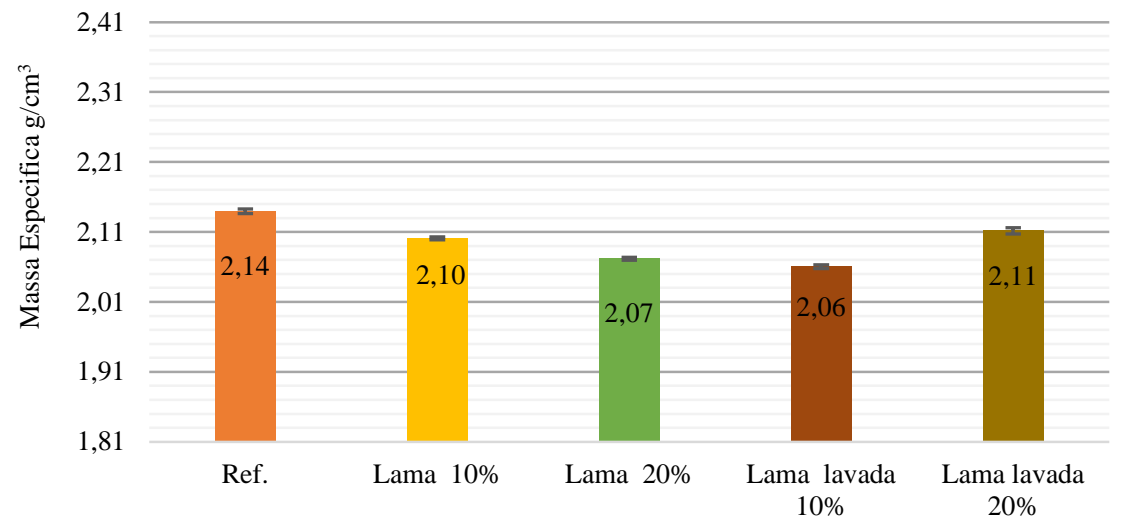

Figura 3: Massa específica dos resíduos de vidro soda-cal-sílica.

Na Figura 4 são apresentados os valores médios de condutividade térmica obtidos para as diferentes argamassas. De acordo com a NBR 15220[2], a condutividade térmica de argamassas de revestimento e de assentamento é $1,15 \mathrm{~W} / \mathrm{mK}$, e a do concreto com brita $1,75 \mathrm{~W} / \mathrm{mK}$. Considerando que a argamassa para fins estruturais é um concreto sem brita e que difere das argamassas de revestimento e assentamento por conter maior massa de cimento, o valor de $1,23 \mathrm{~W} / \mathrm{mK}$ para a argamassa de referência seria adequado com base na regra das misturas e nos valores de condutividade da brita $(2,15$ a 2,79 W/mK) [3, 24]. 


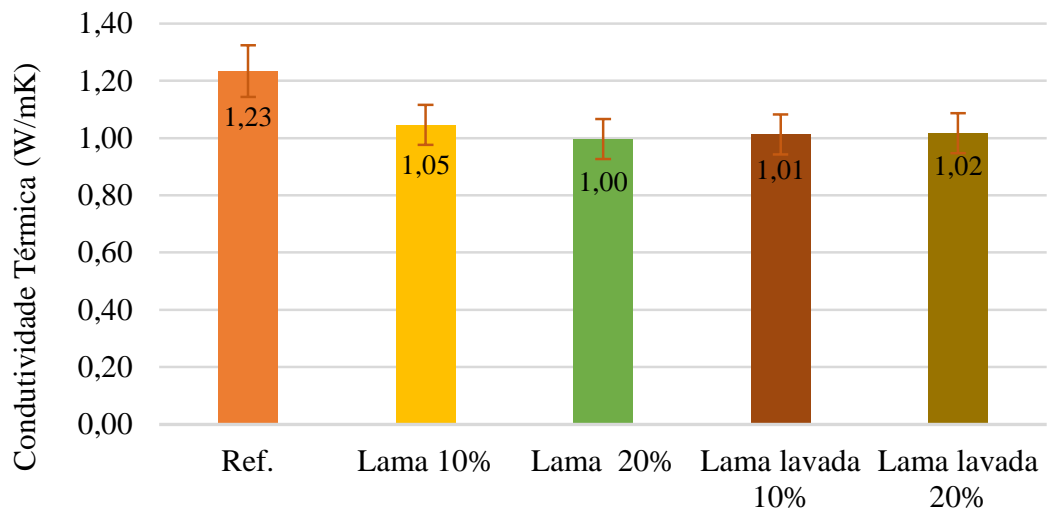

Figura 4: Condutividade térmica das argamassas com e sem lama de vidro.

De forma similar ao observado para massa específica, o teste ANOVA indicou não existirem diferenças significativas entre as condutividades térmicas das argamassas com resíduos de vidro, ou seja, o teor de substituição de cimento Portland por resíduo de vidro, assim como a presença ou não de floculante, não influenciou significativamente a condutividade térmica das amostras. No entanto, o teste estatístico indicou ser significativa a diferença entre essas condutividades e a de referência. Resultados similares foram obtidos por COIMBRA, et al. [25] mostraram que a condutividade térmica de argamassas com resíduos inorgânicos (20 MPa aos 28 dias), naturais (não pozolânicos) e tratados (pozolânicos) é menor que a da amostra padrão (25 MPa aos 28 dias). Como a condutividade depende da natureza do material e da presença de vazios, isto é, varia diretamente com a massa específica [26], os resultados obtidos estariam relacionados, também, à presença de vazios na microestrutura [27].

A emissividade térmica das amostras, determinada pelo método da emissividade conhecida, foi, para todos os materiais, de 0,95, não havendo diferença entre eles. Esse valor se aproxima dos relatados por INCROPERA et.al. [3], sendo para o concreto $(0,88 ; 0,93)$, para o vidro $(0,90 ; 0,95)$, e para argamassas $(0,89$; $0,91)$ [28].

Na Figura 5 são apresentados os perfis típicos de temperatura das faces externas de cada tipo de amostra. A leitura foi feita por termografia infravermelha após o aquecimento das amostras na caixa térmica, considerando a emissividade de 0,95. Verifica-se que existe um tempo de espera, entre 200-250 s, para que o fluxo de calor, gerado pela lâmpada, alcance a face externa da amostra. Após esse tempo, o aumento da temperatura é gradual.

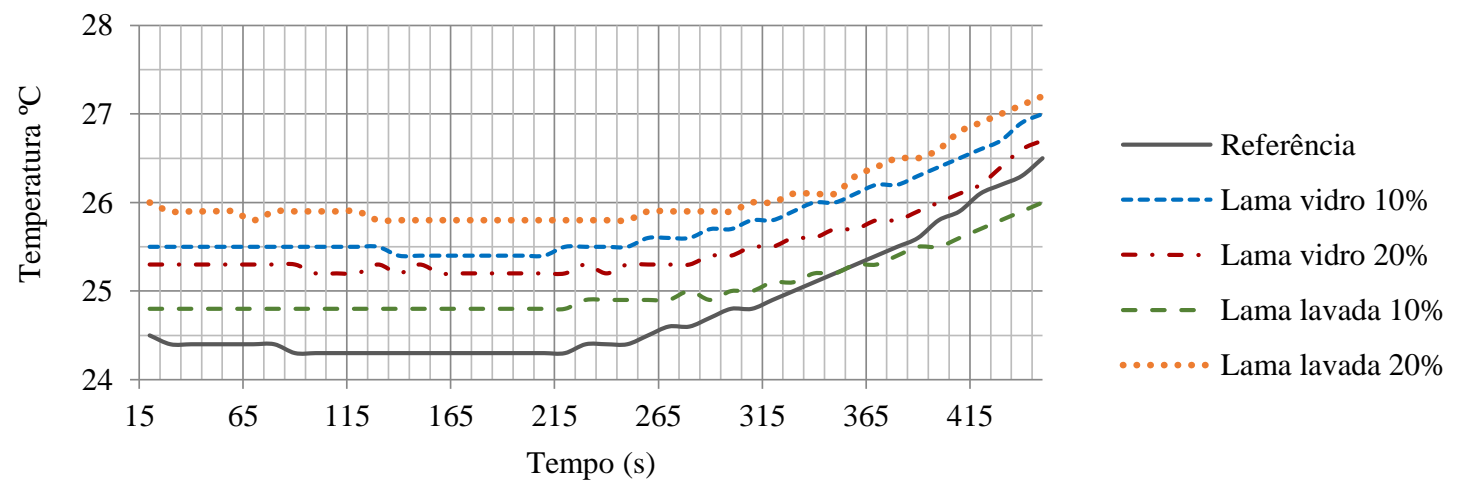

Figura 5: E Perfis de temperatura, obtidos por termografia (face externa das amostras).

Com os dados de condutividade térmica e os perfis de temperatura médios foi possível calcular a difusividade térmica das amostras pelo método do sólido semi-infinito (Equação 1). A Figura 6 exemplifica os dados obtidos para difusividade térmica, considerando as três amostras do material de referência. De forma similar à argamassa de referência, em todas as misturas com vidro os valores da difusividade térmica tendem a diminuir com o tempo de aquecimento e apresentam, uma variação abrupta antes de tenderem à estabilidade. A difusividade térmica característica de cada material foi identificada como aquela que não se modifica com o passar do tempo. 


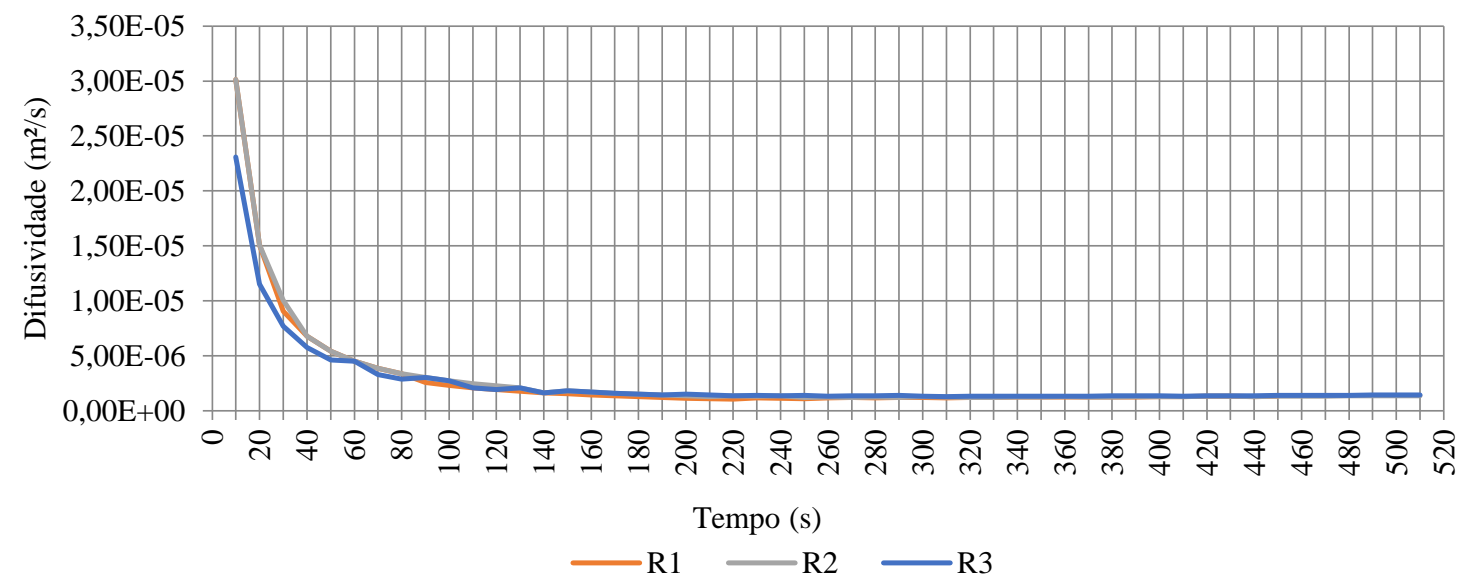

Figura 6: E Evolução da difusividade térmica durante o aquecimento da argamassa de referência.

Na Figura 7 são apresentadas as médias dos valores da difusividade térmica de 3 amostras para cada tipo de material. A amostra de referência apresentou difusividade média de $1,3 \times 10^{-6} \mathrm{~m}^{2} / \mathrm{s}$. Valor compatível aos obtidos para argamassas e concretos convencionais que apresentam difusividade da ordem de $0,7 \times 10^{-6} \mathrm{~m}^{2} / \mathrm{s}(\mathrm{cal}-$ culada) [3] e $0,8-1,5 \times 10^{-6} \mathrm{~m}^{2} / \mathrm{s}$ [29], respectivamente. De acordo com o teste estatístico o uso do resíduo de vidro, nos teores de 10 e $20 \%$ com ou sem floculante, não influenciou a difusividade térmica. Tal resultado estaria relacionado ao fato de que a difusividade térmica depende diretamente da condutividade térmica e inversamente da massa específica e do calor específico a pressão constante. Considerando que o calor específico praticamente não varia com a composição dos materiais cimentícios [3], a diminuição da condutividade devido ao uso da lama (Figura 3) seria compensada com a redução na massa especifica (Figura 2) e, assim, a taxa de transferência de calor se manteria similar à da argamassa de referência, ou seja, a inércia térmica do material não foi influenciada.

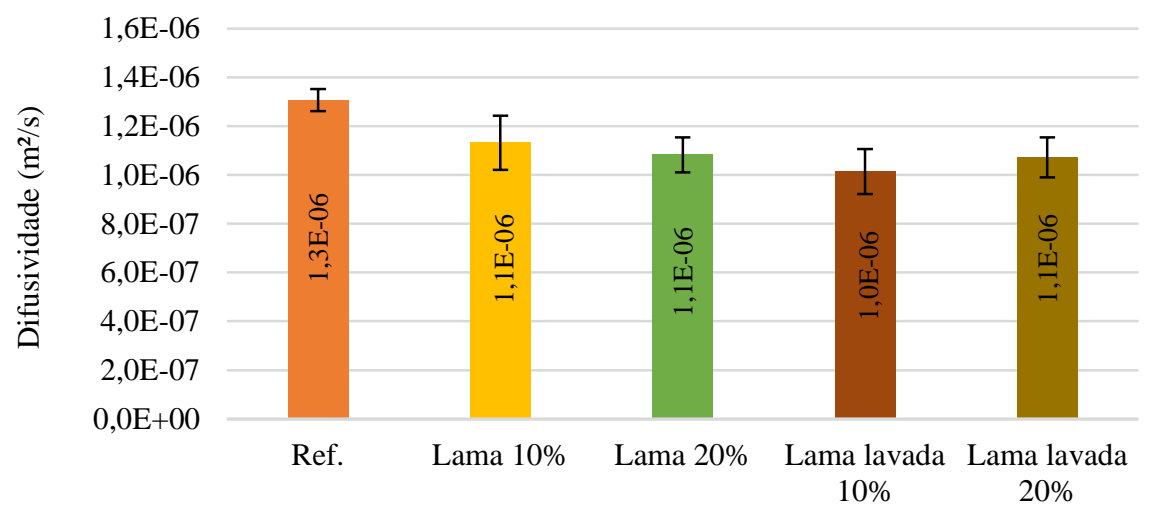

Figura 7: Valores de difusividade térmica das amostras.

\section{CONCLUSÕES}

A análise estatística (ANOVA e teste Tukey) indicaram uma qualidade satisfatória dos dados analisados. O estudo mostra que a substituição do cimento Portland por 10 e $20 \%$ de micropartículas de vidro soda-calsílica, contendo ou não floculante, não compromete a resistência à compressão das argamassas devido às características pozolânicas da lama de vidro. A substituição de $10 \%$ de cimento Portland por resíduo de vidro lavado contribui para um aumento de aproximadamente $8 \%$ na resistência em relação ao valor da amostra de referência. Nos demais casos isto não ocorre, provavelmente, devido aglutinação das partículas promovida pela ação do floculante, e ao efeito de dissolução do cimento que leva a uma menor disponibilidade de hidróxido de cálcio para a ocorrência da reação pozolânica.

Os resultados dos ensaios de massa específica e condutividade térmica não apresentam diferenças significativas entre os valores obtidos para as argamassas contendo resíduos de vidro. No entanto, em relação à amostra de referência, o uso de resíduo, nas condições estudadas, promove uma redução na massa específica de 2 a $4 \%$ e na condutividade térmica de 17 a $18 \%$. A redução da massa específica e da condutividade térmica das argamassas com resíduos de vidro, em relação à de referência, estaria relacionada à existência de vazios nas suas microestruturas, indicando que as reações pozolânicas não ocorreram em toda a sua extensão. 
A termografia infravermelha foi uma técnica auxiliar eficiente para o cálculo da difusividade térmica.

A difusividade térmica das argamassas não é significativamente influenciada pelo uso de 10 e $20 \%$ de lama de vidro, o que se justificaria pelo fato da difusividade variar de forma inversa com a condutividade e massa específica. Isto significa que a inércia térmica, ou seja, a velocidade com que o material reage a mudanças de temperatura não foi comprometida pelo uso de lama de vidro.

Associando-se os resultados referentes à de resistência à compressão e difusividade térmica, pode-se inferir que seria adequado o uso de lama de vidro soda-cal-sílica em substituição a $10 \%$ e $20 \%$ de cimento Portland em argamassas estruturais com vistas ao desempenho térmico do sistema e, principalmente, à redução de impactos ambientais ao se dar uma destinação adequada à lama de vidro e reduzir a retirada de matérias primas para fabricação do cimento Portland.

\section{AGRADECIMENTOS}

This work was supported by the Coordination for the Improvement of Higher Education Personnel (CAPES), the Research Support Foundation of the State of Minas Gerais (FAPEMIG), and the National Council for Scientific and Technological Development (CNPq).

\section{BIBLIOGRAFIA}

[1] FERRARI S., ZANOTTO V. "The thermal performance of walls under actual service conditions: Evaluating the results of climatic chamber tests". Construction and Building Materials. v. 43 pp. 309-316, June 2013.

[2] ASSOCIAÇÃO BRASILEIRA DE NORMAS TÉCNICAS. NBR 15220: Desempenho Térmico de Edificações. Rio de Janeiro, 2005.

[3] INCROPERA, F.P., DEWITT, D.P., BERGMAN, T.L., et al., Principles of Heat and Mass Transfer. 8 ed., John Wiley \& Sons, Global Edition, 2017.

[4] LUCCHI E. "Applications of the infrared thermography in the energy audit of buildings: A review". Renewable and Sustainable Energy Reviews, v. 82 pp. 3077-3090, October 2018.

[5] CIFUENTES, A., MENDIOROZ A., SALAZAR, A. "Simultaneous measurements of the thermal diffusivity and conductivity of thermal insulators using lock-in infrared thermography". International Journal of Thermal Sciences. v. 121, pp. 305-312, Nov. 2017.

[6] BASHEER, C.M. KRISHNAMURTHY C.V., BALASUBRAMANIAM, K. "Hot-rod thermography for in-plane thermal diffusivity measurement”. Measurement, v.103, pp. 235-240, June 2017.

[7] MAROY, K. CARBONEZ, K. STEEMAN, M. et al. "Assessing the thermal performance of insulating glass units with infrared thermography: potential and limitations". Energy Building, v. 138 pp. 175-192, March 2017.

[8] BARBOSA, P. G., RODRIGUES, C., ANDRADE, R. M., et al. "Evaluation of Thermal Diffusivity in Ceramic Blocks Using Infrared Thermography". International Journal of Engineering Technology Research e Management, v. 1, pp. 35-48, Oct., 2018.

[9] PARIS, J.M., ROESSLERA, J.G., FERRARO, C.C., et al. "A review of waste products utilized as supplements to Portland cement in concrete”. Journal of Cleaner Production. v. 121, pp. 1-18. May 2016.

[10] TRENTIN, P. O.; MANICA, J.; VANZETTO, S. C. Substituição parcial de agregado miúdo por resíduo de vidro moído na produção de argamassa. Revista Matéria, v.25, n.1, 2020.

[11] SADIQUL ISLAM, G. M.; RAHMAN, M. H. e KAZI, N. "Waste glass powder as partial replacement of cement for sustainable concrete practice". International Journal of Sustainable Built Environment. V. 6 , pp. 37-44, June 2017.

[12] LEE, H. HANIF, A., USMAN, M., et al. "Performance evaluation of concrete incorporating glass powder and glass sludge wastes as supplementary cementing material”. Journal of Cleaner Production. v. 170, pp. 683-693, January 2018.

[13] IBRAHIM, S., MEAWAD, A. "Assessment of waste packaging glass bottles as supplementary cementitious materials”. Construction and Building Materials, v.182, pp. 451-458, September 2018.

[14] ZACCARON, A., FRIZZO, R.G., ZANONI, E.T. Efeito da adição de resíduo de vidro em massa de cerâmica de alvenaria. Revista Matéria, v.24, n.4, 2019.

[15] SALOMÃO, M.C.F., DIAS, J. F., MARTINS, J. A. L. G., et al., "Estudo sobre a utilização de resíduo de vidro em argamassas cimentícias". In: 52 IBRACON, Congresso Brasileiro do Concreto, Forta-leza, 13 a 
17 de outubro de 2010 .

[16] RAVERDY M, BRIVOT, F., PAILLÉRE A.M., DRON, R. “Appreciation de l'actvite pouzzolanique dos constituents secondaires". In: Proceedings of the 7th International Congress on the Chemistry of Cement, Paris, France, Editions Septima, v. 3, pp. 36-41, 1980.

[17] ASSOCIAÇÃO BRASILEIRA DE NORMAS TÉCNICAS. NBR 12142: Concreto, Determinação da Resistência à Tração na Flexão de Corpos de Prova Prismáticos. Rio de Janeiro, 2010.

[18] PEREIRA, A. M.; SILVA, C. A. R.; QUEIROZ, D. C. A. et al. Estudo das propriedades mecânicas do concreto com adição de cinza de casca de arroz. Revista Matéria, v.20, n.1, pp. 227-238, 2015.

[19] RODIER, L., SAVASTANO, S. "Use of glass powder residue for the elaboration of eco-efficient cementitious materials". Journal of Cleaner Production. v. 184, pp. 333-341, May 2018.

[20] SALES, R.B.C., SALES, F.A., PAZINI, E., et al. "Durability of Mortar Made with Fine Glass Powdered Particles". Advances in Materials Science and Engineering (online), v.17, pp. 1-9, July 2017.

[21] ALIABDO, A. A., ELMOATY, A., ABOSHAMA, A.Y. "Utilization of waste glass powder in the production of cement and concrete". Construction and Building Materials, v. 124, pp. 866-877, October 2016.

[22] SENHADJI, Y., ESCADEILLAS, G., MOULIC, M. et.al. "Influence of natural pozzolan, silica fume and lime-stone fine on strength, acid resistance and microstructure of mortar", Powder Technology, v. 254, pp. 314-323, March 2014.

[23] GHRICI, M. KENAI, S. SAID-MANSOUR M. et. al. Some Engineering Properties of Concrete Containing Natural Pozzolana and Silica Fume. Journal of Asian Architecture and Building Engineering, v. 5:2, pp. 349-354, Julio, 2006.

[24] THAM, M. W., FAZIT, M. R. N. KHALIL, A. H. P. S. et al. "Tensile properties prediction of natural fibre composites using rule of mixtures: A review". Journal of Reinforced Plastics and Composites v. 38 pp. 211-248. November 2018.

[25] COIMBRA, M. A., SANTOS, W. N., MORELLI, M. R. Recuperação de resíduos inorgânicos para a construção civil. Cerâmica v.48 n.306 São Paulo abr./jun. 2002.

[26] ZHANG, Y., SUN, Q., YANG, X. Changes in color and thermal properties of fly ash cement mortar after heat treatment. Construction and Building Materials. v. 165, pp. 72-81, March 2018.

[27] BESSENOUCI, M. Z., BIBI TRIKI, N. E., KHELLADI, et al. The apparent thermal conductivity of pozzolana concrete. Physics Procedia v. 21 pp. 59 - 66, December 2011.

[28] MINIPA DO BRASIL LTDA. Manual de instruções: termômetro infravermelho. http://capitalrefrig.com.br/custom/316/uploads/product/file_544695abad1a9.pdf. (consultado 04/05/2020).

[29] MEHTA, P.K., MONTEIRO, P.J.M. Concreto: microestrutura, propriedades e materiais. 3. ed. São Paulo, Ibracon, 2008.

ORCID

Cristiane do Bom Conselho Sales Alvarenga

Oneida Heiderick Maximo

Isabella Luísa Vieira Aquino Cassimiro

Rosemary do Bom Conselho Sales

Maria Teresa Paulino Aguilar https://orcid.org/0000-0002-5309-2020

https://orcid.org/0000-0002-5001-1010

https://orcid.org/0000-0002-9872-0254

http://orcid.org/0000-0002-9475-0835

https://orcid.org/0000-0002-0121-0881 